\title{
Balancing Gain Narrowing with Self Phase Modulation: 100-fs, 800-nJ from an All-Fiber-Integrated Yb Amplifier
}

\author{
I. Pavlov ${ }^{1,2}$, A. Rybak $^{1,2}$, Ç. Senel ${ }^{1,3}$, and F. Ö. Ilday ${ }^{1}$ \\ ${ }^{1}$ Department of Physics, Bilkent University, 06800 Ankara, Turkey \\ ${ }^{2}$ Institute of Physics, Nauky Av. 46, 680028 Kyiv, Ukraine \\ ${ }^{3}$ TÜBITAK National Metrology Institute (UME), 41470 Kocaeli, Turkey
}

There is much progress in Yb-fiber oscillator-amplifier systems, which enable generation of high-repetitionrate, microjoule energies and sub-picosecond pulse widths $[1,2]$. Given the extremely large total gain factors to reach microjoules starting from nanojoules, which is often in the range of 40-60 dB, due to losses, and the impact of mismatched high-order dispersion as temporal stretching and compression of pulses by large factors (30-40 $\mathrm{dB}$ ) need to be employed. As a result of these challenges, most of the Yb-fiber amplifiers have resulted in pulse durations of several $100 \mathrm{fs}$ or longer. While pulse durations in this range are suited for some applications, there are many cases where 100-fs or shorter pulses in microjoule range are required. Gain narrowing can be effectively countered by self-phase modulation (SPM) [3] by limiting amplification factor in each stage of amplification and through careful optimization of SPM and inversion level along the gain fiber. The conceptual template is readily present in the evolution of the pulse inside the oscillator cavity, where gain factors are often in the 10-50 range per roundtrip. To the extend that the B-integral and the gain distribution along the amplifier can be kept identical to the oscillator by proper scaling of the chirped pulse width and fiber mode area, the original oscillator can be preserved in arbitrary number of amplification stages. Here, we demonstrate a highly fiber-integrated masteroscillator power-amplifier (MOPA) system, from which $\sim 1 \mu \mathrm{J}$ pulses are extracted and externally compressed to $100 \mathrm{fs}$ by arranging amplification in each stage as close as possible to the intra-cavity evolution. To our knowledge, these results are the shortest demonstrated from all-fiber-integrated amplifier at the microjoule level.

The experimental setup is shown schematically in Fig. 1(a). The oscillator with repetition rate of $40 \mathrm{MHz}$, incorporates self-similar pulse evolution in the gain fiber, followed by a segment of anomalous dispersion implemented with diffraction gratings, which impart enough dispersion to switch the sign of chirp to negative, together with mild spectral filtering. The negatively chirped pulses transverse a section of singlemode fiber before completing the loop. While an exact replication of pulse propagation inside the oscillator requires much complexity, the dominant pulse shaping mechanisms could be duplicated in the first and second preamplifiers, where the pulse duration is reduced to $1 \mathrm{MHz}$. In the final stage of amplification, the effects of gain narrowing could not be completely suppressed. However, pulses extracted from the laser system have been compressed to $100 \mathrm{fs}$ without requiring any higher-order dispersion compensation or specialty components.
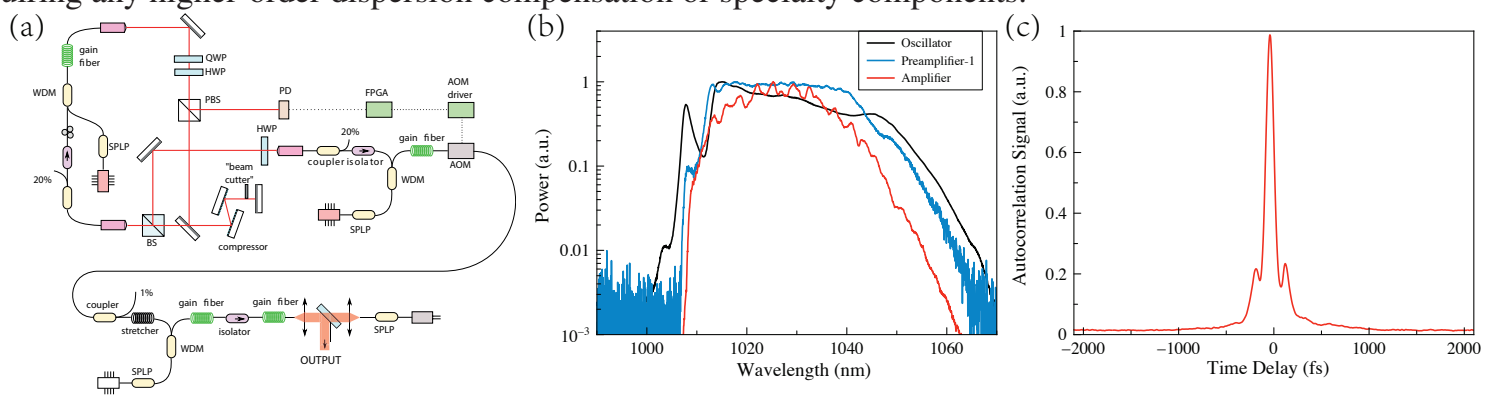

Fig. 1 (a) Schematic of the setup. (b) Optical spectra measured after the oscillator (black trace), the first preamplifier (blue trace) and the final amplifier (red trace). Spectrum after the second preamplifier is virtually identical to that after the first preamplifier. (c) Measured autocorrelation of amplified and dechirped pulses.

In conclusion, we propose a novel scheme for multi-stage fiber amplification of ultrashort pulses, where each stage is designed to mimic pulse amplification and shaping as closely as possible a scaled-version of intra-cavity pulse amplification and scaling, thereby balancing gain narrowing with SPM. While additional work is necessary for full optimisation, as a first experimental demonstration of this approach, we have constructed a highly integrated Yb-doped oscillator-amplifier system, which generates $1.15-\mu \mathrm{J}, 20$-ps pulses at $1030 \mathrm{~nm}$, which are dechirped in a grating compressor, yielding $0.8-\mu \mathrm{J}, 100-$ fs pulses at 1-MHz repetition rate.

\section{References}

[1] J. Limpert, F. Roser, D. N. Schimpf, E. Seise, T. Eidam, S. Hadrich, J. Rothhardt, C. J. Misas, and A. Tünnermann, "High repetition rate gigawatt peak power fiber laser systems: challenges, design, and experiment," IEEE J. Select. Topics Quantum Electron. 15, 159 (2009).

[2] A. Fernandez, K. Jespersen, L. Zhu, L. Grüner-Nielsen, A. Baltuska, A. Galvanauskas, and A. J. Verhoef, "High-fidelity, 160 fs, $5 \mu \mathrm{J}$ pulses from an integrated Yb-fiber laser system with a fiber stretcher matching a simple grating compressor," Opt. Lett. 37, 927 (2012).

[3] M. Hanna, D. Papadopoulos, F. Druon, and P. Georges, "Distributed nonlinear fiber chirped-pulse amplifier system," Opt. Express 17, 10835 (2009). 